\title{
ON CONTINUOUS AND MEASURABLE SELECTIONS AND THE EXISTENCE OF SOLUTIONS OF GENERALIZED DIFFERENTIAL EQUATIONS
}

\author{
HENRY HERMES ${ }^{1}$
}

Abstract. Let $\mathcal{C}\left(B^{n}\right)$ denote the space of nonempty compact subsets of some bounded set $B^{n}$ in Euclidean $n$ dimensional space $E^{n}$, topologized with the Hausdorff metric topology. The existence of a solution to the initial value problem for the generalized differential equation $d x(t) / d t \in R(x(t))$ is shown under the assumption that $R: E^{n} \rightarrow \mathcal{C}\left(B^{n}\right)$ has bounded variation in some neighborhood of the initial value, and under a less restrictive condition on the variation of $R$. Included are continuous and Lipschitz continuous selection results for mappings $Q: E^{1} \rightarrow \mathcal{C}\left(B^{n}\right)$ which are, respectively, of bounded variation and Lipschitz continuous.

Introduction. Let $E^{n}$ denote Euclidean $n$ dimensional space, $B^{n}$ a closed, origin centered ball of radius $b$ in $E^{n}$, and $\mathcal{C}\left(B^{n}\right)$ the metric space of all nonempty compact subsets of $B^{n}$ with the Hausdorff topology. We consider the generalized differential equation

$$
\dot{x}(t) \in R(x(t)), \quad x(0)=x^{0} \in E^{n} \quad(\dot{x}(t)=d x(t) / d t)
$$

where $R: E^{n} \rightarrow \mathfrak{C}\left(B^{n}\right)$ is continuous. A solution of equation (1) is an absolutely continuous function $\phi$ such that $\dot{\phi}(t) \in R(\phi(t))$ for almost all $t$ in some neighborhood of zero, $\phi(0)=x^{0}$; a classical solution of equation (1) is a continuously differentiable function $\phi$ with $\phi(0)=x^{0}$ and $\dot{\phi}(t) \in R(\phi(t))$ for $t$ in some neighborhood of zero.

If $R$ has convex values, the existence of classical solutions of equation (1) may be shown in many ways, see [1] or [2]. Filippov, [3], proves the existence of a solution in the nonautonomous case when the right side is continuous in $t$ and $x$ and satisfies a Lipschitz condition of the form $h\left(R(t, x), R\left(t, x^{\prime}\right)\right) \leqq k(t)\left|x-x^{\prime}\right|$ where $h$ denotes the Hausdorff metric and $k(\cdot)$ is summable. This result can easily be derived by use of a fixed point technique for set valued mappings, see [4]. Filippov also obtains the existence of a classical solution with an additional condition, which is automatically satisfied for the auton-

Received by the editors July 31,1970 .

AMS 1970 subject classifications. Primary 34A99.

Key words and phrases. Generalized differential equation, continuous selections.

${ }^{1}$ This research was partially supported by the Air Force Office of Scientific Research, Office of Aerospace Research, United States Air Force, under AFOSR Grant No. 1243-67.

Copyright (c) 1971, American Mathematical Society 
omous equation. In the present paper the Lipschitz condition is removed and replaced by a weaker condition involving the variation of the set valued map $R$. We show that if $R$ is of bounded variation, a classical solution of equation (1) exists, while under a weaker condition, termed Property A, a solution is shown to exist.

The above-mentioned conditions are pertinent to the question of when a continuous map $Q:[0, t] \rightarrow \mathfrak{C}\left(B^{n}\right)$ admits a continuous selection, i.e. a continuous map $q:[0, T] \rightarrow E^{n}$ with $q(t) \in Q(t)$ for all $t$. It is shown that if $Q$ is of bounded variation, a continuous selection exists. A special case of this is if $Q$ is Lipschitzian in the Hausdorff metric, say with Lipschitz constant $k$, then one may show $Q$ admits a Lipschitz continuous selection $q$, also with Lipschitz constant $k$. These results depend upon the domain of $Q$ being the real line, i.e. they are not true, for example, for mappings $R: E^{3} \rightarrow \mathfrak{C}\left(B^{3}\right)$, see [2].

The following question also naturally arises. Given an equicontinuous family $\left\{Q^{\alpha}\right\}_{\alpha \in A}$ of mappings $Q^{\alpha}:[0, T] \rightarrow \mathfrak{C}\left(B^{n}\right)$, when does there exist a measurable selection $q^{\alpha}$ for each $Q^{\alpha}$ such that the family $\left\{q^{\alpha}\right\}_{\alpha \in A}$ is $\mathfrak{L}_{1}[0, T]$ conditionally compact? A sufficient condition is shown to be, essentially, Property A. If such a family $\left\{q^{\alpha}\right\}_{\alpha \in A}$ could be shown to exist without imposing any additional conditions (such as Property A), the method of proof, employed in the existence theorem given, would lead to an existence theorem for a solution of equation (1) with $R: E^{n} \rightarrow \mathfrak{e}\left(B^{n}\right)$ merely continuous. The existence of a solution, in this case, is still, to the author's knowledge, an open question.

1. Existence of solutions. We assume, throughout, that $R: E^{n}$ $\rightarrow \mathfrak{C}\left(B^{n}\right)$ continuously, where $B^{n}$ is as in the Introduction. $C[0, T]$ will denote the space of continuous ( $n$ vector valued) functions on $[0, T]$ with the uniform norm, and $C_{b}[0, T]$ the compact subset of $C[0, T]$ defined by

$$
C_{b}[0, T]=\left\{x \in C[0, T]: x(0)=x^{0},\left|x(t)-x\left(t^{\prime}\right)\right| \leqq b\left|t-t^{\prime}\right|\right\} .
$$

If $Q \in \mathbb{C}\left(B^{n}\right)$ and $y \in E^{n}$ we use the notation $\rho(y, Q)=\inf \{|y-q|: q \in Q\}$ while for $Q_{1}, Q_{2} \in \mathfrak{e}\left(B^{n}\right), h\left(Q_{1}, Q_{2}\right)$ denotes the Hausdorff distance between these sets.

For $Q:[0, T] \rightarrow \mathfrak{e}\left(B^{n}\right)$, define the variation of $Q$ on the subinterval $[t-\sigma, t], \sigma>0$, denoted $V_{t-\sigma}^{t}(Q)$, as follows. Let $P$ denote a partition of $[t-\sigma, t]$, i.e. a finite collection of points $t-\sigma=t_{0}<t_{1}<\cdots<t_{k+1}$ $=t$, and let $\rho$ denote the set of all such partitions. For the partition $P$, define 
$V_{t-\sigma}^{t}(Q ; P)=\sum_{v=1}^{\nu} h\left(Q\left(t_{\nu+1}\right), Q\left(t_{\nu}\right)\right), \quad V_{t-\sigma}^{t}(Q)=\sup \left\{V_{t-\sigma}^{t}(Q ; P): P \in \odot\right\}$.

Property A. For each $x \in C_{b}[0, T]$,

$$
V_{t-\sigma}^{t}(R(x(\cdot))) \in \mathscr{L}_{1}[0, T] \text { and } \lim _{\sigma \rightarrow 0} \int_{0}^{T} V_{t-\sigma}^{t}(R(x(\cdot))) d t=0
$$

uniformly for $x \in C_{b}[0, T]$. (Assume $x(t)=x(0)$ for $t<0$ so that $V_{t-\sigma}^{t}(R(x(\cdot)))$ is defined for $t-\sigma<0$. This convention will be assumed throughout, when necessary.)

REMARKs. 1. It is clearly possible that the variation $V_{t-\sigma}^{t}(Q)$ is unbounded for some $t$, yet $V_{t-\sigma}^{t}(Q) \in \mathfrak{L}_{1}$. For example, consider the special case of $Q$ a point valued function

$$
\begin{aligned}
q(t) & =(1-t) \sin (1 / 1-t), & & t \neq 1, \\
& =0, & & t=1 .
\end{aligned}
$$

It is well known that $q$ is not of bounded variation in $[0,1]$, yet a simple computation using the property that for $t \neq 1, V_{t-\sigma}^{i}(q)=$ $\int_{t-\sigma}^{t}|\dot{q}(\tau)| d \tau$ shows that $V_{t-\sigma}^{t}(q) \in \mathfrak{L}_{1}[0, T]$, as a function of $t$.

2. If $R$ is Lipschitzian, say $h\left(R(x), R\left(x^{\prime}\right)\right) \leqq K\left|x-x^{\prime}\right|$, clearly Property A is satisfied. Indeed

$$
V_{t-\sigma}^{t}(R(x(\cdot)) ; P)=\sum_{\nu=0}^{k} h\left(R\left(x\left(t_{v+1}\right)\right), R\left(x\left(t_{\nu}\right)\right)\right) \leqq K b \sum_{\nu=0}^{k}\left(t_{\nu+1}-t_{\nu}\right)=K b \sigma
$$

for any $x \in C_{b}[0, T]$.

3. Let $R: E^{n} \rightarrow \mathfrak{C}\left(B^{n}\right)$ continuously and let $S=\left\{y \in E^{n}:\left|y-x^{0}\right| \leqq b T\right\}$. We define the variation of $R$ in $S$, denoted $V(R, S)$, as follows. Let $\lambda$ be any finite collection of points $y^{1}, \cdots, y^{k+1}$ in $S$ such that $\sum_{\nu=1}^{k}\left|y^{\nu+1}-y^{\nu}\right| \leqq b T$ and $\Lambda$ denote the set of all such collections. Let

$$
V(R, S, \lambda)=\sum_{i=1}^{k} h\left(R\left(y^{i+1}\right), R\left(y^{i}\right)\right)
$$

and $V(R, S)$ be defined as $\sup \{V(R, S, \lambda): \lambda \in \Lambda\}$. If $V(R, S)<\infty$ we say $R$ has bounded variation in $S$. Clearly, if $R$ has bounded variation in $S$, then for any $x \in C_{b}[0, T], V_{t-\sigma}^{t}(R(x(\cdot)))<\infty$; in fact $V_{0}^{t}(R(x(\cdot)))$ is finite for all $t \in[0, T]$ and continuous as a function of $t$ (see [5, Theorem 1, p. 223]; the identical proof applies). In this case, $V_{t-\sigma}^{t} R((x(\cdot)))=V_{0}^{t}(R(x(\cdot)))-V_{0}^{t-\sigma}(R(x(\cdot)))$ and hence $\lim _{\sigma \rightarrow 0} V_{t-\sigma}^{t} R(x(\cdot))=0$ for each $x \in C_{b}[0, T]$. It also follows directly from the definition that $V_{t-\sigma}^{t}(R(\cdot))$, considered as a map of $C_{b}[0, T]$ 
$\rightarrow E^{1}$, is continuous, hence since $C_{b}[0, T]$ is compact in $C[0, T]$, one obtains: If $R$ is of bounded variation in $S$, for any $t \in[0, T]$, $\lim _{\sigma \rightarrow 0} V_{t-\sigma}^{t} R(x(\cdot))=0$ uniformly for $x \in C_{b}[0, T]$.

4. For $Q \in \mathfrak{C}\left(B^{n}\right)$, let $Q^{\epsilon}$ denote a closed $\epsilon>0$ neighborhood of $Q$. For each $x \in C_{b}[0, T]$ we consider $R^{\epsilon}(x(\cdot)):[0, T] \rightarrow \mathfrak{C}\left(B^{n}\right)$ (increasing the radius of $B^{n}$ by $\epsilon$ if necessary but retaining the notation $B^{n}$ ). Then $\left\{R^{e}(x(\cdot))\right\}_{x \in C_{b}[0, T]}$ is an equicontinuous family. One may note that in the proof of Theorem 1, which follows, the essential use of Property A is to conclude that one may obtain a measurable (actually continuous) selection $r_{x}$ for each $R^{c}(x(\cdot))$ such that the family $\left\{r_{x}\right\}_{x \in C_{b}[0, T]}$ is $\mathfrak{L}_{1}[0, T]$ conditionally compact.

THEOREM 1. Let $R: E^{n} \rightarrow \mathfrak{e}\left(B^{n}\right)$ continuously. Then:

(a) If $R$ is of bounded variation in the ball $\left\{y \in E^{n}:\left|y-x^{0}\right| \leqq b T\right\}$ equation (1) admits a classical solution on $[0, T]$.

(b) If $R$ satisfies Property A, equation (1) admits a solution on $[0, T]$.

Proof. (In essence, this proof is a modification of the Peano existence proof for ordinary differential equations with continuous right sides.)

The family $\{R(x(\cdot))\}_{x \in C_{b}[0, T]}$ is equicontinuous, where we consider each $R(x(\cdot))$ as a map of $[0, T]$ to $\mathrm{C}\left(B^{n}\right)$. For each $i=1,2, \cdots$, let $\epsilon_{i}>0, \epsilon_{i} \rightarrow 0$ as $i \rightarrow \infty$ and for each $\epsilon_{i}$ let $\delta_{i}>0$ be such that $h\left(R(x(t)), R\left(x\left(t^{\prime}\right)\right)\right)<\epsilon_{i}$ if $\left|t-t^{\prime}\right|<\delta_{i}, x \in C_{b}[0, T]$. We assume, without loss of generality, that $\delta_{i} \rightarrow 0$ as $i \rightarrow \infty$.

Pick $q_{0} \in R\left(x^{0}\right)$. For each $i=1,2, \cdots$, define $r^{i}:\left[-\delta_{1}, T\right] \rightarrow E^{n}$ as follows. Let $r^{i}(t)=q_{0}$ if $-\delta_{1} \leqq t \leqq \delta_{i}$. Define

$$
x^{i}(t)=x^{0}+\int_{0}^{t} r^{i}(\tau) d \tau
$$

for $t \in\left[-\delta_{1}, \delta_{i}\right]$, and choose $q_{1}^{i} \in R\left(x^{i}\left(\delta_{i}\right)\right)$ such that $\left|q_{0}-q_{1}^{i}\right|$ $=\rho\left(q_{0}, R\left(x^{i}\left(\delta_{i}\right)\right)\right)$. We define $r^{i}\left(2 \delta_{i}\right)$ as $q_{1}^{i}$ and extend $r^{i}$ to $\left[-\delta_{1}, 2 \delta_{i}\right]$ as the straight line segment joining $q_{0}$ and $q_{1}^{i}$ on the interval $\left[\delta_{i}, 2 \delta_{i}\right]$. We now extend $x^{i}$ to $\left[-\delta_{1}, 2 \delta_{i}\right]$ by equation (2). Proceeding inductively, if $x^{i}$ is defined on the interval $\left[-\delta_{1}, j \delta_{i}\right]$ choose $q_{j}^{i} \in R\left(x^{i}\left(j \delta_{i}\right)\right)$ such that $\left|q_{j-1}^{i}-q_{j}^{i}\right|=\rho\left(q_{j-1}^{i}, R\left(x^{i}\left(j \delta_{i}\right)\right)\right)$, define $r^{i}\left((j+1) \delta_{i}\right)$ as $q_{j}^{i}$ and extend $r^{i}$ to $\left[-\delta_{1},(j+1) \delta_{i}\right]$ as the straight line segment joining $q_{j-1}^{i}$ and $q_{j}^{i}$ on $\left[j \delta_{i},(j+1) \delta_{i}\right]$. Then extend $x^{i}$ to the interval $\left[-\delta_{1},(j+1) \delta_{i}\right]$ by equation (2). Continue in this manner until each function $r^{i}$ and $x^{i}$ is defined on $\left[-\delta_{1}, T\right]$. We note that $r^{i} \in C\left[-\delta_{1}, T\right]$ while $x^{i} \in C_{b}[0, T]$, when restricted to $[0, T]$. Furthermore, we have: 


$$
\begin{aligned}
\left|r^{i}\left((j+1) \delta_{i}\right)-r^{i}\left(j \delta_{i}\right)\right| & =\left|q_{j}^{i}-q_{j-1}^{i}\right|=\rho\left(q_{j-1}^{i}, R\left(x^{i}\left(j \delta_{i}\right)\right)\right) \\
& \leqq h\left(R\left(x^{i}\left((j-1) \delta_{i}\right)\right), R\left(x^{i}\left(j \delta_{i}\right)\right)\right)<\epsilon_{i} .
\end{aligned}
$$

(ii) Given any $t \in[0, T]$ there exists an integer $j$ such that $\left|j \delta_{i}-t\right|$ $<\delta_{i}$. Then

$$
\begin{aligned}
\rho\left(r^{i}(t), R\left(x^{i}(t)\right)\right) \leqq & \left|r^{i}(t)-r^{i}\left(j \delta_{i}\right)\right|+\rho\left(r^{i}\left(j \delta_{i}\right), R\left(x^{i}\left(j \delta_{i}\right)\right)\right) \\
& +h\left(R\left(x^{i}\left(j \delta_{i}\right)\right), R\left(x^{i}(t)\right)\right) \\
\leqq & 3 \epsilon_{i} .
\end{aligned}
$$

(iii) For any $t, t^{\prime} \in[0, T]$, let $j, j^{\prime}$ be integers such that $\left|t-j \delta_{i}\right|$ $<\delta_{i},\left|t^{\prime}-j^{\prime} \delta_{i}\right|<\delta_{i}$. Assume, with no loss of generality, that $j^{\prime}>j$. Then

$$
\begin{aligned}
\left|r^{i}(t)-r^{i}\left(t^{\prime}\right)\right| \leqq & \rho\left(r^{i}(t), R\left(x^{i}\left(j \delta_{i}\right)\right)\right) \\
& +\sum_{\nu=j}^{j^{\prime}-1} h\left(R\left(x^{i}\left((\nu+1) \delta_{i}\right)\right), R\left(x^{i}\left(\nu \delta_{i}\right)\right)\right) \\
& +\rho\left(r^{i}\left(t^{\prime}\right), R\left(x^{i}\left(j^{\prime} \delta_{i}\right)\right)\right) \\
\leqq & 4 \epsilon_{i}+\sum_{\nu=j}^{j^{\prime}-1} h\left(R\left(x^{i}\left((\nu+1) \delta_{i}\right)\right), R\left(x^{i}\left(\nu \delta_{i}\right)\right)\right)
\end{aligned}
$$

We are now in a position to prove conclusion (a) of Theorem 1. If $R$ is of bounded variation, it follows from Remark 3 that $\left\{r^{i}(\cdot)\right\}$ is a bounded equicontinuous sequence in $C[0, T]$. Indeed, given any $\epsilon>0$, choose $i^{*}$ sufficiently large so that $i \geqq i^{*}$ implies $4 \epsilon_{i}<\epsilon / 2$; now choose $\delta>0$ such that for $0 \leqq \sigma<\delta, V_{t-\sigma}^{t} R(x(\cdot))<\epsilon / 2$ uniformly for $x \in C_{b}[0, T]$. Then from (iii), above, it follows that if $i \geqq i^{*},\left|t-t^{\prime}\right|<\delta$ then $\left|r^{i}(t)-r^{i}\left(t^{\prime}\right)\right|<\epsilon$, as desired. Thus $\left\{r^{i}\right\}$ has a uniformly convergent subsequence $\left\{r^{i k}(\cdot)\right\}$ which converges, say, to $r$. Then $\left\{x^{i k}(\cdot)\right\}$ has a uniformly convergent subsequence, say converging to $x$ $\in C_{b}[0, T]$. From (ii) we see $r(t) \in R(x(t))$, since $R(x(t))$ is closed, while taking a limit in equation (2) gives $x(t)=x^{0}+\int_{0}^{t} r(\tau) d \tau$. Thus $\dot{x}(t)=r(t) \in R(x(t))$, for all $t ; x(0)=x^{0}$, showing $x$ is a classical solution of (1).

To obtain conclusion (b) of Theorem 1, if $R$ satisfies Property A we find from (iii) that

$$
\int_{0}^{T}\left|r^{i}(t)-r^{i}(t-\sigma)\right| d t \leqq 4 \epsilon_{i} T+\int_{0}^{T} V_{t-\sigma}^{t}\left(R\left(x^{i}(\cdot)\right)\right) d t .
$$

Now given any $\epsilon>0$ we can use Property $A$ to choose a $\sigma_{0}>0$ such 
$\int_{0}^{T} V_{i-\sigma}^{t}\left(R\left(x^{i}(\cdot)\right)\right) d t<\epsilon / 2$ if $0 \leqq \sigma \leqq \sigma_{0}$, all $i=1,2, \cdots$. Next choose $i^{*}$ sufficiently large so that for $i \geqq i^{*}, 4 \epsilon_{i} T<\epsilon / 2$. Then for $i \geqq i^{*}$, $0 \leqq \sigma \leqq \sigma_{0}, \int_{0}^{T}\left|r^{i}(t)-r^{i}(t-\sigma)\right| d t<\epsilon$. By [6, Theorem 20, p. 298], the sequence $\left\{r^{i}\right\}$ is $\mathfrak{L}_{1}[0, T]$ conditionally compact. Thus it has an $\mathfrak{L}_{1}[0, T]$ convergent subsequence, $\left\{r^{i k}\right\}$, which converges, say, to $r$. Again $\left\{x^{i_{k}}\right\}$ has a uniformly convergent subsequence, converging say, to $x \in C_{b}[0, T]$. From (ii) we see $r(t) \in R(x(t))$ almost everywhere, while taking a limit in equation (2) gives $x(t)=x^{0}+\int_{0}^{t} r(\tau) d \tau$ or $\dot{x}(t)=r(t) \in R(x(t))$ almost everywhere, $x(0)=x^{0}$, showing that $x$ is a solution (not necessarily a classical solution) of equation (1).

2. Continuous selections for continuous maps $Q:[0, T] \rightarrow \mathfrak{C}\left(B^{n}\right)$. Much recent work, [7], [8], has been done to show the existence of measurable selections (or selections which are Baire mappings) for continuous (or lower semicontinuous) mappings $F: X \rightarrow 2^{Y}$ where $X$ is a metric space, $Y$ a complete, separable, metric space, and $2^{Y}$ the set of nonempty closed subsets of $Y$ with the Hausdorff topology. In general, one cannot expect more than a Baire selector, indeed if $Q:[0, T] \rightarrow \mathfrak{C}\left(B^{2}\right)$ continuously, many examples exist to show that $Q$ need not admit a continuous selection, see, for example, [2]. Even if one imposes further conditions, such as a Lipschitz condition, on $Q: E^{3} \rightarrow \mathfrak{C}\left(B^{3}\right)$, one may give a counterexample to the existence of a continuous selection, see [2]. However, for the special case when the domain of $Q$ is $E^{1}$, we may obtain the following

Theorem 2. Let $Q:[0, T] \rightarrow \mathfrak{C}\left(B^{n}\right)$ continuously, Then

(a) If $Q$ has bounded variation in $[0, T]$, i.e. if $V_{0}^{T}(Q)<\infty$, then $Q$ admits a continuous selection $r$.

(b) If $Q$ satisfies a Lipschitz condition of the form $h\left(Q(t), Q\left(t^{\prime}\right)\right)$ $\leqq K\left|t-t^{\prime}\right|$ then $Q$ admits a Lipschitz continuous selection $r$ satisfying $\left|r(t)-r\left(t^{\prime}\right)\right| \leqq K\left|t-t^{\prime}\right|$, i.e. the same Lipschitz constant.

Proof. For each positive integer $k$, consider the points $0, T / k$, $2 T / k, \cdots, T$. Choose $q_{0}^{\boldsymbol{k}} \in Q(0) ; q_{1}^{\boldsymbol{k}} \in Q(T / k)$ and such that $\left|q_{0}^{\boldsymbol{k}}-q_{1}^{\boldsymbol{k}}\right|$ $=\rho\left(q_{0}^{k}, Q(T / k)\right)$, and inductively $q_{j}^{k} \in Q(j T / k)$ and such that $\left|q_{j-1}^{k}-q_{j}^{k}\right|$ $=\rho\left(q_{j-1}^{k}, Q(j T / k)\right)$. Define $r^{k}:[0, T] \rightarrow E^{n}$ as the polygonal arc joining the points $q_{j}^{k}, j=0, \cdots, k$. Then

(i) For any $t \in[0, T]$ and any $k$, there exists an integer $j=j(k)$ such that $|t-i T / k|<T / k$. Assume, with no loss of generality, that $t \in[(j-1) T / k, j T / k]$. Then

$$
\begin{aligned}
\rho\left(r^{k}(t), Q(t)\right) & \leqq\left|r^{k}(t)-r^{k}(j T / k)\right|+\rho\left(r^{k}(j T / k), Q(t)\right) \\
& \leqq h(Q((j-1) T / k), Q(j T / k))+h(Q(j T / k), Q(t)) .
\end{aligned}
$$


(ii) For $t, t^{\prime} \in[0, T]$ and any $k$, let $j, j^{\prime}$ be integers such that $|t-j T / k|<T / k,\left|t^{\prime}-j^{\prime} T / k\right|<T / k$. Then

$$
\begin{aligned}
\left|r^{k}(t)-r^{k}\left(t^{\prime}\right)\right| \leqq & \left|r^{k}(t)-r^{k}(j T / k)\right| \\
& +\sum_{\nu=j}^{j^{\prime}-1}\left|r^{k}((\nu+1) T / k)-r^{k}(\nu T / k)\right| \\
& +\left|r^{k}\left(j^{\prime} T / k\right)-r^{k}\left(t^{\prime}\right)\right| \\
\leqq & h(Q(t), Q(j T / k))+\sum_{\nu=j}^{j^{\prime}-1} h(Q((\nu+1) T / k), Q(\nu T / k)) \\
& +h\left(Q\left(t^{\prime}\right), Q\left(j^{\prime} T / k\right)\right) .
\end{aligned}
$$

Now, to show part (a), we first show that $\left\{r^{k}\right\}_{k=1,2} \ldots$ is equicontinuous. Given any $\epsilon>0$ first choose $k^{*}$ sufficiently large so that for $k \geqq k^{*}, h\left(Q\left(t_{1}\right), Q\left(t_{2}\right)\right)<\epsilon / 3$ if $\left|t_{1}-t_{2}\right|<T / k^{*}$. Next, since $Q$ is of bounded variation, $V_{0}^{t}(Q)$ is continuous as a function of $t$ on $[0, T]$, hence uniformly continuous, and we can choose a $\delta>0$ such that $V_{a}^{b}(Q)<\epsilon / 3$ if $|a-b|<\delta$. Since $\left|j T / k-j^{\prime} T / k\right| \leqq\left|t-t^{\prime}\right|+2 T / k$ if $k>4 T / \delta$ and $\left|t-t^{\prime}\right|<\delta / 2, V_{j T / k}^{j T / k}(Q)<\epsilon / 3$. Then from (ii) we have: for $k \geqq \max \left(4 T / \delta, k^{*}\right)$ and $\left|t-t^{\prime}\right|<\delta,\left|r^{k}\left(t_{0}\right)-r^{k}\left(t^{\prime}\right)\right|<\epsilon / 3+\epsilon / 3+\epsilon / 3=\epsilon$ and equicontinuity is shown. Clearly the sequence $\left\{r^{k}\right\}$ is bounded hence it has a uniformly convergent subsequence, say converging to $r \in C[0, T]$. Let $t \in[0, T]$ and $j(k)$ be an integer such that $|t-j(k) T / k|$ $<T / k$, i.e. $j(k) T / k \rightarrow t$ as $k \rightarrow \infty$. From (i), and the fact that the set $Q(t)$ is closed, it follows that $r(t) \in Q(t)$, i.e. $r$ is the desired continuous selection.

To show part (b), we assume, without loss of generality in (ii), that $t \leqq j T / k<\cdots<j^{\prime} T / k \leqq t^{\prime}$. Then utilizing the Lipschitz condition for $Q$, (ii) becomes

$$
\begin{aligned}
& \left|r^{k}(t)-r^{k}\left(t^{\prime}\right)\right| \\
& \quad \leqq K\left[((j T / k)-t)+\sum_{\nu=j}^{j^{\prime}-1}(((\nu+1) T / k)-\nu T / k)+\left(t^{\prime}-j^{\prime} T / k\right)\right] \\
& \quad=K\left|t^{\prime}-t\right| .
\end{aligned}
$$

Thus $\left\{r^{k}\right\}$ is equicontinuous, bounded, and now has a subsequence converging uniformly to, say, $r \in C[0, T]$ and $r$ satisfies $\left|r(t)-r\left(t^{\prime}\right)\right|$ $\leqq K\left|t-t^{\prime}\right|$. Again, from (i), we conclude $r(t) \in Q(t)$ and $r$ is the desired selection. 


\section{REFERENCES}

1. H. Hermes, The generalized differential equation $\dot{x} \in R(t, x)$, Advances in Math. 4 (1970), 149-169. MR 40 \#5939.

2. - Existence and properties of solutions of $\dot{x} \in R(t, x)$, Studies in Appl. Math., no. 5, SIAM Publications, 1969, pp. 188-193.

3. A. F. Filippov, Classical solutions of differential equations with multivalued right-hand side, Vestnik Moskov. Univ. Ser. I Mat. Meh. 22 (1967), no. 3, 16-26; English transl., SIAM J. Control 5 (1967), 609-621. MR 35 \#5683; MR 36 \#4047.

4. $\mathrm{H}$. Hermes, $\mathrm{O}$ the structure of attainable sets for generalized differential equations and control systems, J. Differential Equations 9 (1971), 141-154.

5. I. P. Natanson, Theory of functions of a real variable, Ungar, New York, 1955. MR 16, 804.

6. N. Dunford and J. T. Schwartz, Linear operators. I: General theory, Pure and Appl. Math., vol. 7, Interscience, New York, 1958. MR 22 \#8302.

7. K. Kuratowski and C. Ryll-Nardzewski, $A$ general theorem on selectors, Bull. Acad. Polon. Sci., Sér. Sci. Math. Astronom. Phys. 13 (1965), 397-403. MR 32 \#6421.

8. M. M. Čoban, Multivalued mappings and Borel sets, Dokl. Akad. Nauk SSSR 182 (1968), 514-517 = Soviet Math. Dokl. 9 (1968), 1175-1178. MR 38 \#5185.

University of Colorado, Boulder, Colorado 80302 